\title{
Cyber Attack Detection in Platoon-Based Vehicular Networked Control Systems
}

\author{
Eman Mousavinejad*, Fuwen Yang*, Qing-Long Han ${ }^{\dagger}$, Quanwei Qiu* and Ljubo Vlacic* \\ ${ }^{*}$ Griffith School of Engineering, Griffith University, Gold Coast, QLD 4222, Australia \\ Email: \{eman.mousavinejad, quanwei.qiu\}@griffithuni.edu.au, \{fuwen.yang, l.vlacic\}@griffith.edu.au \\ ${ }^{\dagger}$ School of Software and Electrical Engineering, Swinburne University of Technology \\ John Street, Hawthorn, Melbourne, VIC 3122, Australia \\ Email: qhan@swin.edu.au
}

\begin{abstract}
This paper is concerned with cyber attack detection problem in a platoon-based vehicular networked control system. In such a system, the information among vehicles is transmitted through a shared wireless communication network and also each vehicle has access to its own information measured by local sensors. These kind of systems are highly vulnerable to cyber attacks and therefore, cyber-security issues need to be properly addressed to ensure the safety of the systems. Among various cyber-security aspects, reliable attack detection is of utmost importance as the ability to detect cyber attacks in a timely manner can reduce the damage to the systems. Therefore, we present a cyber attack detection algorithm that is capable of detecting attacks violating both measurements and control command data. This algorithm is based on an ellipsoidal set-membership filtering approach which consists of two sets: prediction ellipsoid set and an estimation ellipsoid set calculated through updating the prediction ellipsoid set with the measurement data. The detection method depends on the existence of intersection between these two sets computed by the filter. Simulation results for some possible cyber attacks are provided to demonstrate the effectiveness of the proposed method.
\end{abstract}

Index Terms-Cyber attack detection, platoon-based vehicles, Set-membership filtering, networked control systems.

\section{INTRODUCTION}

A platoon-based vehicular networked control system (NCS) is an advanced automated method of driving a group of vehicles with some common interests on a road. This kind of system not only improves road safety, highway efficiency and capacity but also reduces traffic congestion [1]. Generally, an effective way to increase road capacity is forming vehicles into a platoon where the speed of each following vehicle is automatically adjusted to reduce inter-vehicle distance without compromising safety. To this end, one of the most developed Intelligent Transportation System (ITS) technology is so-called Cooperative Adaptive Cruise control (CACC) which is an extension of Adaptive Cruise Control (ACC) [1].

A general diagram of a single vehicle forming a platoonbased vehicular system is shown in Fig. 1 whose objective is to reach a given velocity or acceleration of the leader vehicle in the platoon while ensuring a spacing policy among the other vehicles. To satisfy this aim, each vehicle must be equipped with additional on-board sensors (radar, camera, lidar) in order to monitor the predecessor's states acquired by the ACC and also with a shared wireless communication

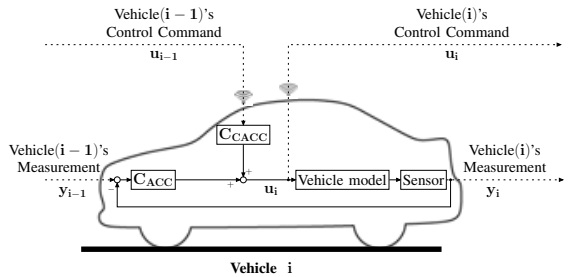

Fig. 1. Schematic of a single vehicle in a platoon

network where inter-vehicle data used by the CACC in each vehicle are exchanged so that the platoon can reach its common interest. Therefore, a vehicle platoon system can be represented as an NCS where the vehicles are considered as mobile nodes connected together via wireless channels and a shared communication network as shown in Fig. 2.

As depicted in Fig. 2, the control commands and sensor measurement outputs are transmitted through wireless communication channels between two consecutive vehicles. Moreover, since in this framework the attack detection system is considered to be at the remote site, any of the system's control commands and its sensors measurement outputs are transmitted to the remote site via a shared communication network. Therefore, the content of these signals can be modified by both cyber attacks and networked-induced phenomena such as induced-delay, quantization, and packet dropout. In this study, however, the communication network will be assumed to be free of any networked-induced communication constraint in order to just focus on the cyber attack detection problem.

Since the key aspect of platoon-based vehicular NCSs is

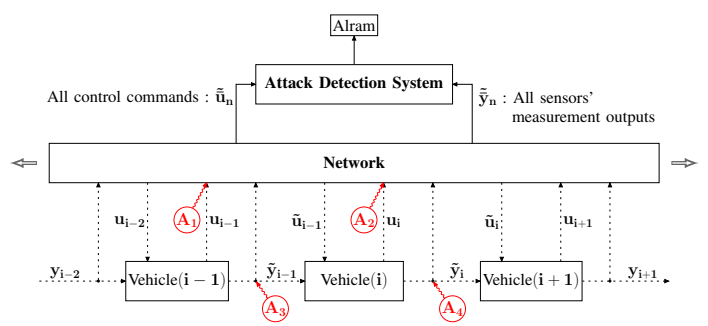

Fig. 2. Schematic of a general platoon-based vehicular NCS with some possible attack points 
that the required information among vehicles is exchanged through a wireless communication network in addition to those gathered from on-board sensors, the cooperative platoon-based driving pattern is more vulnerable to cyber attacks which may result in traffic chaos even car crash on road. Attacks on an NCS can be practically considered either attacks directly on physical components (physical attack), i.e. sensors and controllers, or attacks on the wireless communication channels that transmit data to receivers (cyber attack). In this paper, it will be considered that only the communication channels transmitting data are under cyber attacks. As a result, if there is a cyber attack violating any of the system's control commands $u_{i}$ and/or any of its sensors measurement outputs $y_{i}$, they will be modified to $\tilde{u}_{i}$ and $\tilde{y}_{i}$, respectively. The propagation of the output of the attack detection system throughout the control system is not considered in this paper. Instead, it has just been considered as an alarm signal.

Recently, considerable effort has been devoted to develop network-aware modeling of a platoon-based vehicular system and improve string stability of such a system. To mention a few, the practical challenges of a vehicular platoon are reviewed in [2] in which the importance of robust wireless communication is highlighted. The effects of communication delays on string stability is investigated in [3]-[5]. Longitudinal platoon control via communication channels with packet loss is addressed in [6]-[10]. The impact of network delay integrated with packet loss on the platoon stability is studied in [11], [12]. Although there have been a majority of results in the literature investigating the network-induced phenomena in platoon-based vehicular NCSs, little attention has been paid to the problem of cyber attack detection in this research area. As discussed in [1], [13], cyber security in vehicular network is one of the critical issues in commercializing vehicle platooning in highways.

Motivated by the discussion above, it is the objective of this paper to design an algorithm by means of set-membership filtering techniques to detect cyber attacks into platoon-based vehicular NCSs. A recursive convex optimization algorithm is proposed to compute the state estimate ellipsoid that guarantees to contain the true state for an attack-free system. This algorithm estimates the state in two steps: a prediction step and a measurement update step. Then, two sub-algorithms are provided to detect cyber attacks in platoon-based vehicular NCSs. The idea to detect cyber attacks introduced in the subalgorithms depends on the existence of intersection between the prediction ellipsoid set and the estimation ellipsoid set calculated by updating the prediction ellipsoid set with the measurement data. If there exists no intersection between the prediction ellipsoid set and the estimation ellipsoid set updated with the previous measurement output, control signal is compromised by attacks; the sensor (measurement output) signal is violated by attacks if there is no intersection between the prediction ellipsoid set and the estimation ellipsoid set updated at the current time instant.

\section{Problem Statement and Formulation}

Various models for vehicle dynamics have been considered in the study of longitudinal control of platoons. In this paper, the dynamics model presented in [5] with the same notations is adopted. In this model, each vehicle must follow its predecessor while maintaining a desired distance considering a constant headway time spacing policy. The desired spacing, $d_{r, i}$, between the front bumper of $i$-th vehicle to its predecessor's, $(i-1)$-vehicle, rear bumper is

$$
d_{r, i}=r_{i}+h_{d, i} v_{i}
$$

where $r_{i}$ is the desired distance at standstill, $h_{d, i}$ is the headway time constant which is the time takes for the $i$ th vehicle to arrive at same position as its predecessor, and $v_{i}$ is the vehicle velocity. The actual distance between two consecutive vehicles is

$$
d_{i}=q_{i-1}-\left(q_{i}+L_{i}\right)
$$

where $q_{i}$ is the absolute position of the $i$-th vehicle in global coordinates in the longitudinal direction and $L_{i}$ is the length of the $i$-th vehicle. The local vehicle following control objective is regulating the error

$$
e_{i}=d_{i}-d_{r, i}
$$

to zero asymptotically.

As discussed in [5], the vehicle following controller consists of two controllers: ACC and CACC. The ACC is a feedback PD-type controller that acts on locally sensed data. These data consist of the relative distance $\left(q_{i}-q_{i-1}\right)$, and relative velocity $\left(v_{i}-v_{i-1}\right)$ which are measured through sensors mounted at the front of each vehicle, and local measurements of velocity $v_{i}$ and acceleration $a_{i}$ of each vehicle. Therefore, the control command signal can be written as

$$
u_{f b, i}=k_{p, i} e_{i}+k_{d, i}\left(v_{i-1}-v_{i}-h_{d, i} a_{i}\right)
$$

The ACC satisfies the objective of the vehicle following controller, i.e. regulating $e_{i}$ to zero, if the vehicle platoon travels with a constant speed in steady state. To improve vehicle following and string stability performance, a CACC is introduced as an addition to the ACC in a feedforward fashion. The control signal of the CACC is

$$
\dot{u}_{f f, i}=-h_{d, i}^{-1} u_{f f, i}+h_{d, i}^{-1} u_{i-1}
$$

where $u_{i-1}$ is the desired acceleration of the predecessor vehicle and it is transmitted through a shared wireless communication network between two consecutive vehicles. Consequently, the total control command $u_{i}$ is given by

$$
u_{i}=u_{f b, i}+u_{f f, i}
$$

Defining the $i$-th vehicle's state variables as $x_{i}=$ $\left[\begin{array}{llll}e_{i} & v_{i} & a_{i} & u_{f f, i}\end{array}\right]^{T}$, the linearized state-space representation of longitudinal dynamics of the $i$-th vehicle in a platoon of $n$ vehicles can be represented by

$$
\dot{x}_{i}=A_{i, i} x_{i}+A_{i, i-1} x_{i-1}+B_{s, i} u_{i}+B_{c, i} u_{i-1} \quad i \in \mathbb{N}_{[2, n]}
$$


where

$$
\begin{gathered}
A_{i, i}=\left[\begin{array}{cccc}
0 & -1 & -h_{d, i} & 0 \\
0 & 0 & 1 & 0 \\
0 & 0 & -\eta_{i}^{-1} & 0 \\
0 & 0 & 0 & -h_{d, i}^{-1}
\end{array}\right], \quad B_{s, i}=\left[\begin{array}{c}
0 \\
0 \\
\eta_{i}^{-1} \\
0
\end{array}\right] \\
A_{i, i-1}=\left[\begin{array}{llll}
0 & 1 & 0 & 0 \\
0 & 0 & 0 & 0 \\
0 & 0 & 0 & 0 \\
0 & 0 & 0 & 0
\end{array}\right], \quad B_{c, i}=\left[\begin{array}{c}
0 \\
0 \\
0 \\
h_{d, i}^{-1}
\end{array}\right]
\end{gathered}
$$

where $\eta_{i}$ is a parameter representing the internal actuator dynamics; $u_{i}$ is the desired acceleration for the $i$-th vehicle; $B_{s, i}$ is the input vector corresponding to the total control command $u_{i}$ to the $i$-th vehicle and $B_{c, i}$ is the input vector for the additional CACC input $u_{i-1}$ received by the $i$-th vehicle through the wireless network.

The total control command (6) can be written in terms of the state variables as

$$
\begin{aligned}
u_{i} & =u_{f b, i}+u_{f f, i} \quad i \in \mathbb{N}_{[1, n]} \\
& =K_{i, i-1} x_{i-1}+K_{i, i} x_{i} \\
K_{i, i-1}= & {\left[\begin{array}{c}
0 \\
k_{d, i} \\
0 \\
0
\end{array}\right]^{T}, \quad K_{i, i}=\left[\begin{array}{c}
k_{p, i} \\
-k_{d, i} \\
-k_{d, i} h_{d, i} \\
v
\end{array}\right]^{T} }
\end{aligned}
$$

where $v=1$ corresponds to an operational CACC, and $v=0$ gives only ACC.

A reference vehicle model is used to generate the trajectory for the lead vehicle supposing there is no preceding vehicle in front of it. The dynamics of the reference vehicle denoted by $i=0$ is described as

$$
\begin{gathered}
\dot{x}_{0}=A_{0} x_{0}+B_{s, 0} u_{r} \\
A_{0}=\left[\begin{array}{cccc}
0 & 0 & 0 & 0 \\
0 & 0 & 1 & 0 \\
0 & 0 & -\eta_{0}^{-1} & 0 \\
0 & 0 & 0 & 0
\end{array}\right], \quad B_{s, 0}=\left[\begin{array}{c}
0 \\
0 \\
\eta_{0}^{-1} \\
0
\end{array}\right]
\end{gathered}
$$

where $x_{0}=\left[\begin{array}{llll}e_{0} & v_{0} & a_{0} & u_{f f, 0}\end{array}\right]^{T}$, and $u_{r}$ is the reference acceleration profile. In (9), there are two redundant states, i.e. $e_{0}, u_{f f, 0}$, since the state variables are chosen in accordance with those for the upcoming vehicle in the platoon in (7).

It should be pointed out that the CACC reference input, $u_{r}$ is locally available to the lead vehicle $(i=1)$ as it is generated by this vehicle. Therefore, the state-space representation of the platoon-based vehicular NCS including all $n$ vehicles can be represented as

$$
\dot{\bar{x}}_{n}=\bar{A}_{n} \bar{x}_{n}+\bar{B}_{s, n} \bar{u}_{n}+\bar{B}_{c, n} \bar{u}_{n}+B_{r} u_{r}
$$

where

$$
\begin{gathered}
\bar{A}_{n}=\left[\begin{array}{ccccc}
A_{0} & 0 & 0 & \ldots & 0 \\
A_{1,0} & A_{1,1} & 0 & \ldots & 0 \\
0 & A_{2,1} & A_{2,2} & \ldots & 0 \\
\vdots & & \ddots & \ddots & \vdots \\
0 & \ldots & 0 & A_{n, n-1} & A_{n, n}
\end{array}\right], \quad B_{r}=\left[\begin{array}{c}
B_{s, 0} \\
B_{c, 1} \\
0 \\
\vdots \\
0
\end{array}\right] \\
\bar{B}_{s, n}=\left[\begin{array}{cccc}
0 & \ldots & \ldots & 0 \\
B_{s, 1} & 0 & \ldots & 0 \\
0 & B_{s, 2} & 0 & \vdots \\
\vdots & & \ddots & 0 \\
0 & \ldots & 0 & B_{s, n}
\end{array}\right], \quad \bar{B}_{c, n}=\left[\begin{array}{cccc}
0 & \ldots & 0 & 0 \\
0 & \ldots & 0 & 0 \\
B_{c, 2} & \ldots & 0 & 0 \\
\vdots & \ddots & \vdots & \vdots \\
0 & \ldots & B_{c, n} & 0
\end{array}\right]
\end{gathered}
$$

$$
\bar{x}_{n}=\left[\begin{array}{lllll}
x_{0}^{T} & x_{1}^{T} & x_{2}^{T} & \ldots & x_{n}^{T}
\end{array}\right]^{T}, \quad \bar{u}_{n}=\left[\begin{array}{llll}
u_{1} & u_{2} & \ldots & u_{n}
\end{array}\right]^{T}
$$

The control command in (8) can now be written in the form of

$$
\bar{u}_{n}=\bar{K}_{n} \bar{x}_{n}
$$

with

$$
\bar{K}_{n}=\left[\begin{array}{ccccc}
K_{1,0} & K_{1,1} & 0 & \ldots & 0 \\
0 & K_{2,1} & K_{2,2} & \ldots & 0 \\
\vdots & & \ddots & \ddots & \vdots \\
0 & \ldots & & K_{n, n-1} & K_{n, n}
\end{array}\right]
$$

As discussed in [5], in the interconnected vehicle platoon model (10), the control command, i.e. $\bar{u}_{n}$ is kept separate since it is used in deferent ways by the host vehicle, i.e. through direct measurement in ACC or through wireless communication in CACC, respectively. Also, in (10), the control command used by ACC can be expressed as a state feedback control law. Therefore, (10) is now reformulated in a compact form as

$$
\begin{aligned}
\dot{\bar{x}}_{n} & =\left(\bar{A}_{n}+\bar{B}_{s, n} \bar{K}_{n}\right) \bar{x}_{n}+\bar{B}_{c, n} \bar{u}_{n}+B_{r} u_{r} \\
& =A_{\bar{x}_{n}}^{A C C} \bar{x}_{n}+\bar{B}_{c, n} \bar{u}_{n}+B_{r} u_{r}
\end{aligned}
$$

where $A_{\bar{x}_{n}}^{A C C}=\bar{A}_{n}+\bar{B}_{s, n} \bar{K}_{n}$, and $u_{r}$ is the exogenous input.

Now, the discrete-time representation of (12) must be derived to be used for an attack detection algorithm. The discretetime model at the sampling instants $t_{k}=k T, k \in \mathbb{N}$ can be expressed as

$$
\bar{x}_{n, k+1}=A_{n, k} \bar{x}_{n, k}+B_{c, n, k} \bar{u}_{n, k}+B_{r, k} u_{r, k}
$$

with

$$
\begin{gathered}
A_{n, k}=e^{A_{\overline{\bar{x}}_{n}}^{A C C} T}, \quad B_{c, n, k}=\int_{0}^{T} e^{A_{\overline{\bar{x}}_{n}}^{A C C} s} d s \bar{B}_{c, n} \\
B_{r, k}=\int_{0}^{T} e^{A_{\bar{x}_{n}}^{A C C} s} d s B_{r}
\end{gathered}
$$

Considering process and measurement noises in an unknown-but-bounded (UBB) sequence, the discrete-time platoon-based vehicular NCS model (13) can be described as

$$
\begin{aligned}
\bar{x}_{n, k+1} & =A_{n, k} \bar{x}_{n, k}+B_{c, n, k} \bar{u}_{n, k}+B_{r, k} u_{r, k}+F_{n, k} w_{k} \\
\bar{y}_{n, k} & =C_{n, k} \bar{x}_{n, k}+D_{n, k} v_{k}
\end{aligned}
$$

where $\bar{y}_{n, k} \in \mathbb{R}^{m}$ is the measurement outputs gathered from all sensors; $A_{n, k}, B_{c, n, k}, B_{r, k}, F_{n, k}, C_{n, k}$ and $D_{n, k}$ are known matrices with appropriate dimensions; $w_{k} \in \mathbb{R}^{r}$ and $v_{k} \in \mathbb{R}^{p}$ are the UBB process and measurement noises, respectively, which are confined to the following specified ellipsoidal sets

$$
\begin{aligned}
\mathcal{W}_{k} & :=\left\{w_{k}: w_{k}^{T} Q_{k}^{-1} w_{k} \leq 1\right\} \\
\mathcal{V}_{k} & :=\left\{v_{k}: v_{k}^{T} R_{k}^{-1} v_{k} \leq 1\right\}
\end{aligned}
$$

where $Q_{k}=Q_{k}^{T} \succ 0$ and $R_{k}=R_{k}^{T} \succ 0$ are known matrices with compatible dimensions.

The initial state $\bar{x}_{n, 0}$ is assumed to belong to a given ellipsoidal set $\mathcal{X}_{0 \mid 0}\left(\hat{\bar{x}}_{n, 0 \mid 0}, E_{0 \mid 0}\right)$

$$
\mathcal{X}_{0 \mid 0}:=\left\{\bar{x}_{n, 0}:\left(\bar{x}_{n, 0}-\hat{\bar{x}}_{n, 0 \mid 0}\right)^{T} P_{0 \mid 0}^{-1}\left(\bar{x}_{n, 0}-\hat{\bar{x}}_{n, 0 \mid 0}\right) \leq 1\right\}
$$




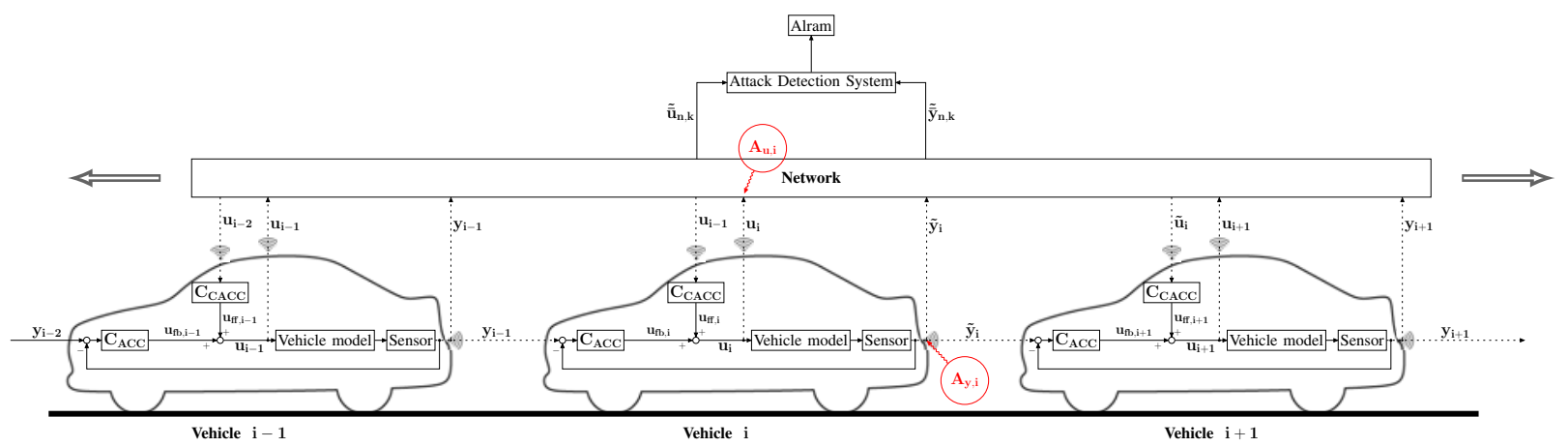

Fig. 3. Platoon-based vehicular NCS framework with an attack detection system and cyber attacks on the i-th vehicle

where $\hat{\bar{x}}_{n, 0 \mid 0}$ is an estimate of $\bar{x}_{n, 0}$, which is assumed to be given, $P_{0 \mid 0}=P_{0 \mid 0}^{T} \succ 0$ is a known matrix and $P_{0 \mid 0}=$ $E_{0 \mid 0} E_{0 \mid 0}^{T}$.

\section{Attack Detection Using Set-Membership FILTERING}

let us consider the framework of a platoon-based vehicular NCS as shown in Fig. 3. In this section, a set-membership filtering method is designed for the proposed cyber attack detection problem.

\section{A. Prediction Ellipsoid Set}

The prediction filter is considered in the form of

$$
\hat{\bar{x}}_{n, k+1 \mid k}=G_{k} \hat{\bar{x}}_{n, k \mid k}+B_{c, n, k} \bar{u}_{n, k}+B_{r, k} u_{r, k}
$$

where $\hat{\bar{x}}_{n, k \mid k}$ is an estimation of the state $\bar{x}_{n, k} ; G_{k}$ is the filter parameter to be determined.

In the first step, a previous state estimation ellipsoid at instant $k$ is assumed to be known and belong to a set $\mathcal{X}_{k \mid k}\left(\hat{\bar{x}}_{n, k \mid k}, E_{k \mid k}\right)$ with the centre $\hat{\bar{x}}_{n, k \mid k}$ and the shape matrix $P_{k \mid k}$, i.e.

$$
\bar{x}_{n, k}=\hat{\bar{x}}_{n, k \mid k}+E_{k \mid k} z
$$

where $E_{k \mid k} E_{k \mid k}^{T}=P_{k \mid k}$, and for some $z$ such that $\|z\| \leq 1$.

Now, the goal is to obtain the prediction ellipsoid set $\mathcal{X}_{k+1 \mid k}$ :

$$
\begin{aligned}
& \mathcal{X}_{k+1 \mid k}:=\left\{\bar{x}_{n, k+1}:\right. \\
&\left.\left(\bar{x}_{n, k+1}-\hat{\bar{x}}_{n, k+1 \mid k}\right)^{T} P_{k+1 \mid k}^{-1}\left(\bar{x}_{n, k+1}-\hat{\bar{x}}_{n, k+1 \mid k}\right) \leq 1\right\}
\end{aligned}
$$

Theorem 1: For the system (14)-(15), suppose that the state $\bar{x}_{n, k}$ belongs to its state estimation ellipsoid (20). Then the one-step ahead state $\bar{x}_{n, k+1}$ resides in its state prediction ellipsoid (21) for any value of the system noises belong to their specified sets, if there exist $P_{k+1 \mid k}>0, G_{k}, \tau_{1} \geq 0$, and $\tau_{2} \geq 0$ such that the following recursive matrix inequality holds.

$$
\left[\begin{array}{cc}
-P_{k+1 \mid k} & \Pi_{1, k} \\
\Pi_{1, k}^{T} & -\Theta_{1}\left(\tau_{1}, \tau_{2}\right)
\end{array}\right] \leq 0
$$

where

$$
\begin{aligned}
& \Pi_{1, k}=\left[\begin{array}{lll}
\left(A_{n, k}-G_{k}\right) \hat{\bar{x}}_{n, k \mid k} & A_{n, k} E_{k \mid k} & F_{n, k}
\end{array}\right] \\
& \Theta_{1}\left(\tau_{1}, \tau_{2}\right)=\operatorname{diag}\left(1-\tau_{1}-\tau_{2}, \tau_{1} I, \tau_{2} Q_{k}^{-1}\right)
\end{aligned}
$$

Corollary 1: Consider the system (14)-(15) and the prediction filter (19). As the size of an ellipsoid can be measured by means of the sum of squared semiaxes lengths, which is given by $\operatorname{Tr}(P)^{1}$, the convex optimization approach is applied to determine an optimal ellipsoid. $P_{k+1 \mid k}$ is obtained by solving the following optimization problem:

$$
\underset{P_{k+1 \mid k}>0, G_{k}, \tau_{1} \geq 0, \tau_{2} \geq 0}{\operatorname{minimize}} \operatorname{Tr}\left(P_{k+1 \mid k}\right)
$$

subject to (22)

\section{B. Estimation Ellipsoid Set}

A filter based on the current measurement is considered for the system (14)-(15), which is in the form of

$$
\hat{\bar{x}}_{n, k+1 \mid k+1}=\hat{\bar{x}}_{n, k+1 \mid k}+L_{k+1}\left(\bar{y}_{n, k+1}-\hat{\bar{y}}_{n, k+1 \mid k}\right)
$$

where $L_{k+1}$ is the filter parameter to be determined.

At the measurement update step, the prediction ellipsoid set $\mathcal{X}_{k+1 \mid k}$ is given by (21) for the state $\bar{x}_{n, k+1}$, which can be written as

$$
\bar{x}_{n, k+1}=\hat{\bar{x}}_{n, k+1 \mid k}+E_{k+1 \mid k} z
$$

where $E_{k+1 \mid k} E_{k+1 \mid k}^{T}=P_{k+1 \mid k}$, and for some $z$ such that $\|z\| \leq 1$.

Our objective is to update this prediction set with the one yielding from the current measurement $\bar{y}_{n, k+1}$. Thus, the updated ellipsoid set should satisfy the condition

$$
\left(\bar{x}_{n, k+1}-\hat{\bar{x}}_{n, k+1 \mid k+1}\right)^{T} P_{k+1 \mid k+1}^{-1}\left(\bar{x}_{n, k+1}-\hat{\bar{x}}_{n, k+1 \mid k+1}\right) \leq 1
$$

whenever the equality

$$
\bar{y}_{n, k+1}=C_{k+1} \hat{\bar{x}}_{n, k+1 \mid k}+C_{n, k+1} E_{k+1 \mid k} z+D_{n, k+1} v_{k+1}
$$

holds for some $\|z\| \leq 1$ and $v_{k+1} \in \mathcal{V}_{k+1}$.

Theorem 2: For the system (14)-(15), if the state $\bar{x}_{n, k+1}$ belongs to its state prediction ellipsoid (25), then the updated state $\bar{x}_{n, k+1}$ resides in its state estimation ellipsoid (26), if there exist $P_{k+1 \mid k+1}>0, L_{k+1}, \tau_{3} \geq 0, \tau_{4} \geq 0$, and $N_{k+1}$ such that the following recursive matrix inequality holds.

$$
\left[\begin{array}{cc}
-P_{k+1 \mid k+1} & \Pi_{2} \\
\Pi_{2}^{T} & \Theta_{3}\left(\tau_{3}, \tau_{4}, \tau_{5}, \hat{\bar{x}}_{n, k+1 \mid k}, N_{k+1}\right)
\end{array}\right] \leq 0
$$

\footnotetext{
${ }^{1} \operatorname{Tr}(P)$ denotes trace of $P$.
} 
where

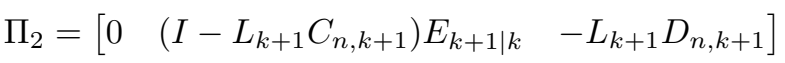

$$
\begin{aligned}
& \Theta_{3}\left(\tau_{3}, \tau_{4}, \hat{\bar{x}}_{n, k+1 \mid k}, N_{k+1}\right)=-\Theta_{2}\left(\tau_{3}, \tau_{4}\right)+ \\
& N_{k+1}^{T} \Pi_{y}\left(\hat{\bar{x}}_{n, k+1 \mid k}\right)+\Pi_{y}^{T}\left(\hat{\bar{x}}_{n, k+1 \mid k}\right) N_{k+1} \\
& \Pi_{y}\left(\hat{\bar{x}}_{n, k+1 \mid k}\right)=\left[C_{n, k+1} \hat{\bar{x}}_{n, k+1 \mid k}-\bar{y}_{n, k+1} \quad C_{n, k+1} E_{k+1 \mid k}\right. \\
& \left.D_{n, k+1}\right] \\
& \Theta_{2}\left(\tau_{3}, \tau_{4}\right)=\operatorname{diag}\left(1-\tau_{3}-\tau_{4}, \tau_{3} I, \tau_{4} R_{k+1}^{-1}\right)
\end{aligned}
$$

Corollary 2: Consider the system (14)-(15) and filter (24). Applying the convex optimization approach, an optimal ellipsoid can be determined. $P_{k+1 \mid k+1}$ is obtained by solving the following optimization problem:

$$
\begin{aligned}
& \underset{P_{k+1 \mid k+1}>0, L_{k+1}, N_{k+1}, \tau_{3} \geq 0, \tau_{4} \geq 0}{\operatorname{minimize}} \operatorname{Tr}\left(P_{k+1 \mid k+1}\right) \\
& \text { subject to (28) }
\end{aligned}
$$

\section{Attach Detection Algorithm}

The algorithm that recursively computes the prediction and estimation ellipsoid sets is summarized as Algorithm 1. Based on the intersection between these two ellipsoid sets, two subalgorithms are defined to detect attacks that violate the control signal and the sensor measurement.

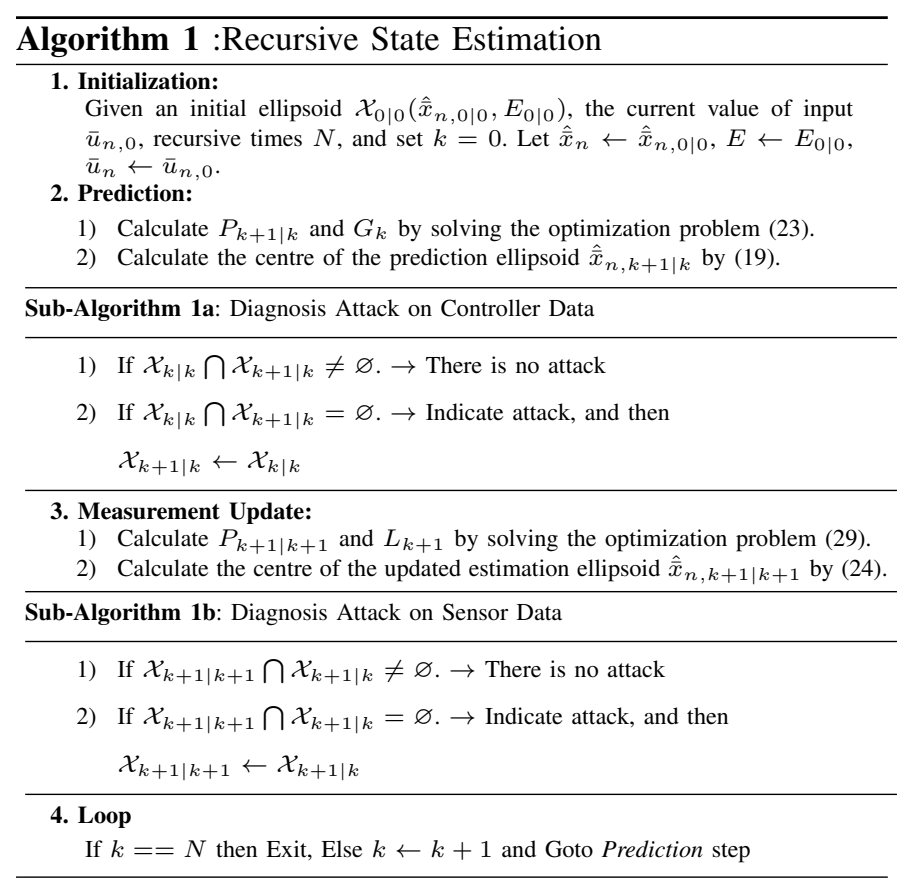

\section{Results AND Discussion}

In this section, the simulation results are obtained under Matlab 8.6 with YALMIP and the solver Sdpt 3 during 250 sampling steps with the sample time $T=0.1 \mathrm{sec}$. A vehicle string formed by one leader $(i=1)$ and two followers $(i=2,3)$ are considered to demonstrate the effectiveness of the proposed attack detection algorithm. The string desired acceleration introduced by the reference vehicle in the string $(i=0)$ is in the form of

$$
u_{r, k}=\left\{\begin{array}{ll}
1 \mathrm{~m} / \mathrm{sec}^{2}, & 50<k<150 \\
0, & \text { otherwise }
\end{array} .\right.
$$

The parameters for each vehicle in the string are considered the same, which corresponds to a homogeneous vehicle string. These parameters are set as $\eta_{i}=0.1, h_{d, i}=0.3, k_{p, i}=$ 0.25 , and $k_{d, i}=0.5 . w_{k}$ and $v_{k}$ are chosen as $0.02 \sin (2 k)$ and $0.02 \sin (20 k)$, respectively. $Q_{k}=1$ and $R_{k}=1$. The initial state is set as $\bar{x}_{n, 0}=\left[\begin{array}{llll}x_{0,0}^{T} & x_{1,0}^{T} & \ldots & x_{n, 0}^{T}\end{array}\right]^{T}$ with $x_{n, 0}=$

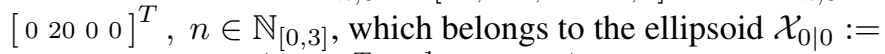
$\left\{\bar{x}_{n, 0}:\left(\bar{x}_{n, 0}-\hat{\bar{x}}_{n, 0 \mid 0}\right)^{T} P_{0 \mid 0}^{-1}\left(\bar{x}_{n, 0}-\hat{\bar{x}}_{n, 0 \mid 0}\right) \leq 1\right\}$, where $\hat{\bar{x}}_{n, 0 \mid 0}=\left[\begin{array}{llll}\hat{\bar{x}}_{0,0}^{T} & \hat{x}_{1,0}^{T} & \ldots & \hat{x}_{n, 0}^{T}\end{array}\right]^{T}$ with $\hat{\bar{x}}_{n, 0}=\left[\begin{array}{llll}0 & 18.96 & 0 & 0\end{array}\right]^{T}, n \in$ $\mathbb{N}_{[0,3]}$, and $P_{0 \mid 0}=100 \cdot \mathcal{I}_{16,16}$.

To perform a successful attack by an attacker, it is assumed that an attacker has knowledge of the accurate values of the control signals $\bar{u}_{n, k}$ and the sensor measurement outputs $\bar{y}_{n, k}$ in real time, and he is also capable of violating the integrity of the measurement outputs and control signals and modifying their true values to arbitrary ones.

\section{A. Replay Attack on Sensors Data}

The replay attack consists of two phases: (1) the attacker starts to record sequences of data from sensors' communication channels without entering any input to the system. (2) the attacker replays the recorded data to the system [14].

An attacker can record the sensor measurement data from $k_{o}$ till $k_{r}$ with the window size of $\tau=k_{r}-k_{o}$ in the first phase. Then, in the second phase, the attacker replays the recorded data to the system from $k=k_{r}+1$ till the end on the attack at $k=k_{f}$. The replay attack set can be modeled as

$$
\mathcal{A}_{y}=\left\{A_{y, i}: A_{y, i}=y_{i, k-\tau}-y_{i, k}, i \in \mathbb{N}_{[1, n]}\right\}
$$

Thus, the sensor measurement output affected by the attack is

$$
\tilde{\bar{y}}_{n, k}=\left\{\tilde{y}_{i, k}: \tilde{y}_{i, k}=y_{i, k}+\Gamma^{y} A_{y, i}, i \in \mathbb{N}_{[1, n]}\right\}
$$

where $\Gamma^{y}$ belongs to the boolean set indicating whether a replay attack is performed $\left(\Gamma^{y}=1\right)$ or not $\left(\Gamma^{y}=0\right)$.

In the simulation, it is assumed that the attacker records the sensor's data of the second vehicle $(i=2)$ from step $k=80$ till $k=99$ and then replaces the sensor's data with the recorded data from step $k=100$ to $k=119$. Fig. $4 \mathrm{a}$ demonstrates the sequence of the intersection between the prediction and the updated estimation ellipsoid sets during the simulation time.

As the replay attack starts at $k=100$, the prediction ellipsoid set is calculated from the sensor measurement data obtained at $k=99$ when there is no attack. However, the estimation ellipsoid set is updated with the current sensor measurement data at $k=100$. Therefore, it is expected from the Sub-algorithm $1 b$ that there must be no intersection between the prediction ellipsoid set and the estimation ellipsoid set updated at the current time instant when the attack starts at $k=100$, i.e. $\chi_{100 \mid 99} \cap \chi_{100 \mid 100}=\varnothing$. 

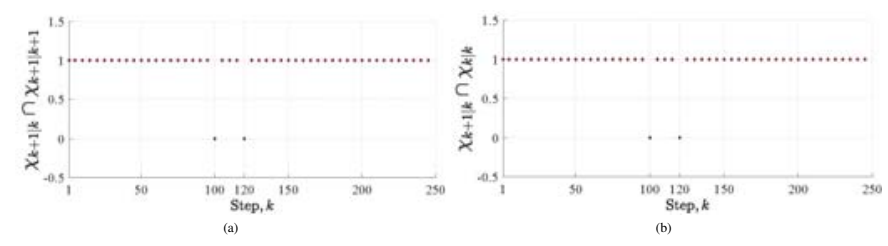

Fig. 4. (a) Replay attack on vehicle $(i=2)$ sensor data, intersection $\chi_{k+1 \mid k}$ and $\chi_{k+1 \mid k+1}$ (b) Bias injection attack on control input $u_{1}$, intersection $\chi_{k+1 \mid k}$ and $\chi_{k \mid k}$. (" 1 ": there exists intersection, " 0 ": there is no intersection)

Once the attack finishes at $k=120$, the prediction ellipsoid set is based on the sensor measurement data at $k=119$ which is under the attack, while the estimation ellipsoid set is updated with the current sensor measurement data at $k=120$ on which there is no attack. As a result, there is no intersection between these two sets, i.e. $\chi_{120 \mid 119} \cap \chi_{120 \mid 120}=\varnothing$, as shown in Fig. 4a. During steps $k=1$ till $k=99$ and $k=121$ till $k=250$, the system is free of the attack, so from the Sub-algorithm $1 b$ it can be concluded that there exists intersection between the two sets as illustrated in Fig. 4a.

\section{B. Bias Injection Attack on Control Commands Data}

In the bias injection attack, the attacker injects a constant bias in the system [14]. The bias injection attack set on the control signal can be modeled as

$$
\mathcal{A}_{u}=\left\{A_{u, i}: A_{u, i}=\delta_{i}, i \in \mathbb{N}_{[1, n]}\right\}
$$

where $\delta_{i}$ is a constant value injected by the attacker. Therefore, the control signal's data affected by the attack is

$$
\tilde{\bar{u}}_{n, k}=\left\{\tilde{u}_{i, k}: \tilde{u}_{i, k}=u_{i, k}+\Gamma^{u} A_{u, i}, i \in \mathbb{N}_{[1, n]}\right\}
$$

where $\Gamma^{u}$ belongs to the boolean set indicating whether a bias injection attack is performed $\left(\Gamma^{u}=1\right)$ or not $\left(\Gamma^{u}=0\right)$.

In the simulation, it is considered that the attacker carries out a bias injection attack on the control signal $u_{1}$, which is sent by the vehicle $i=1$ and received by the vehicle $i=2$, via targeting the wireless communication channel between these vehicles. The attack vector is modeled as $\mathcal{A}_{u}=\left[\begin{array}{lll}4 & 0 & 0\end{array}\right]^{T}$ from step $k=100$ to $k=119$.

As the bias injection attack starts at $k=100$, the centre of the prediction ellipsoid set is affected by the attack; however the attack does not affect the previous updated estimation ellipsoid set since it is based on the prediction set at step $k=99$. Therefore, from the Sub-algorithm la, there must be no intersection between the prediction ellipsoid set and the previous updated estimation ellipsoid set when the attack starts at $k=100$, i.e. $\chi_{101 \mid 100} \cap \chi_{100 \mid 100}=\varnothing$, as depicted in Fig. 4 b.

Once the attack finishes at $k=120$, the centre of the prediction ellipsoid set is not further affected by the attack; however the attack affects the previous updated estimation ellipsoid set since it is based on the prediction set at step $k=119$. Therefore, it is expected from the Sub-algorithm la that there must be no intersection between the prediction ellipsoid set and the previous updated estimation ellipsoid set when the attack finishes at $k=120$, i.e. $\chi_{121 \mid 120} \cap \chi_{120 \mid 120}=\varnothing$, as depicted in Fig. 4b.

\section{CONCLUSION}

This paper has presented a cyber attack detection algorithm based on the ellipsoidal set-membership filtering approach in a platoon-based vehicular NCS. The algorithm consists of a prediction ellipsoid set and an estimation ellipsoid set updated with the measurement output. The attack detection method relies on the intersection of the two sets. Cyber attacks on control signals can be detected if the prediction ellipsoid set does not have intersection with the estimation set updated with the measurement data received at the previous time instant. Cyber attacks on sensors measurements can be detected if there is no intersection between the prediction ellipsoid set and the estimation ellipsoid set updated with the current measurement data.

\section{REFERENCES}

[1] D. Jia, K. Lu, J. Wang, X. Zhang, and X. Shen, "A survey on platoonbased vehicular cyber-physical systems," IEEE Commun. Surveys Tuts., vol. 18, no. 1, pp. 263-284, Firstquarter 2016.

[2] K. C. Dey, L. Yan, X. Wang, Y. Wang, H. Shen, M. Chowdhury, L. Yu, C. Qiu, and V. Soundararaj, "A review of communication, driver characteristics, and controls aspects of cooperative adaptive cruise control (cacc)," IEEE Trans. Intell. Transp. Syst., vol. 17, no. 2, pp. 491509, Feb 2016.

[3] J. K. Hedrick, Y. Chen, and S. Mahal, "Optimized vehicle control/communication interaction in an automated highway system," California Partners for Advances Transit and Highways, BERKELEY, Tech. Rep. UCB-ITS-PRR-2001-29, Oct. 2001.

[4] X. Liu, A. Goldsmith, S. S. Mahal, and J. K. Hedrick, "Effects of communication delay on string stability in vehicle platoons," in Proc. 2001 IEEE Intelligent Transportation Systems, CA, USA, Aug. 2001, pp. 625-630.

[5] S. nc, J. Ploeg, N. van de Wouw, and H. Nijmeijer, "Cooperative adaptive cruise control: Network-aware analysis of string stability," IEEE Trans. Intell. Transp. Syst., vol. 15, no. 4, pp. 1527-1537, Aug 2014.

[6] P. Seiler and R. Sengupta, "Analysis of communication losses in vehicle control problems," in Proc. the 2001 American Control Conference, VA, USA, Jun. 2001, pp. 1491-1496.

[7] P. Seiler and R. Sengupta, "An $h_{\infty}$ approach to networked control," IEEE Trans. Autom. Control, vol. 50, no. 3, pp. 356-364, March 2005.

[8] R. Teo, D. M. Stipanovic, and C. J. Tomlin, "Decentralized spacing control of a string of multiple vehicles over lossy datalinks," IEEE Trans. Control Syst. Technol., vol. 18, no. 2, pp. 469-473, March 2010.

[9] C. Lei, E. M. van Eenennaam, W. K. Wolterink, G. Karagiannis, G. Heijenk, and J. Ploeg, "Impact of packet loss on cacc string stability performance," in Proc. 11th International Conference on ITS Telecommunications, Aug 2011, pp. 381-386.

[10] G. Guo and S. Wen, "Communication scheduling and control of a platoon of vehicles in vanets," IEEE Trans. Intell. Transp. Syst., vol. 17, no. 6, pp. 1551-1563, June 2016.

[11] G. Guo and W. Yue, "Hierarchical platoon control with heterogeneous information feedback," IET Control Theory Applications, vol. 5, no. 15, pp. 1766-1781, October 2011

[12] S. Santini, A. Salvi, A. S. Valente, A. Pescap, M. Segata, and R. L. Cigno, "A consensus-based approach for platooning with inter-vehicular communications," in Proc. IEEE Conference on Computer Communications (INFOCOM), Kowloon, Hong Kong, April 2015, pp. 1158-1166.

[13] J. Petit and S. E. Shladover, "Potential cyberattacks on automated vehicles," IEEE Trans. Intell. Transp. Syst., vol. 16, no. 2, pp. 546-556, April 2015.

[14] A. Teixeira, D. Pérez, H. Sandberg, and K. H. Johansson, "Attack models and scenarios for networked control systems," in Proc. the 1st International Conference on High Confidence Networked Systems, Beijing, China, Apr. 2012, pp. 55-64.

[15] A. A. Kurzhanskiy and P. Varaiya, "Ellipsoidal toolbox," EECS Department, University of California, Berkeley, Tech. Rep., May 2006. 